\title{
Contrast-Associated Acute Kidney Injury (CA-AKI) in Children: Special Considerations
}

\author{
Martin Windpessl, M.D. ${ }^{1}$ \\ Andreas Kronbichler, M.D., Ph.D. ${ }^{2}$
}

Department of Internal Medicine IV (Nephrology Section) ${ }^{1}$, Klinikum WelsGrieskirchen, Grieskirchner Straße 42, 4600 Wels, Austria, Department of Internal Medicine IV (Nephrology and Hypertension) ${ }^{2}$, Medical University Innsbruck, Anichstraße 35, 6020 Innsbruck, Austria

Corresponding author: Martin WindpessI, M.D. Department of Internal Medicine IV (Nephrology Section), Klinikum WelsGrieskirchen, Grieskirchner Straße 42, 4600 Wels, Austria

Tel: 0043-724-241-592194

E-mail:martin.windpess@@klinikum-wegr.at

Andreas Kronbichler, M.D. Ph.D.

Department of Internal Medicine IV (Nephrology and Hypertension), Anichstraße 35, 6020 Innsbruck, Austria Tel: 0043-512-504-81338

E-mail: andreas.kronbichler@i-med.ac.at

Received: 29 August 2019

Revised: 4 October 2019

Accepted: 8 October 2019
This is an open-access article distributed under the terms of the Creative Commons Attribution Non-Commercial License (http:// creativecommons.org/licenses/by-nc/4.0/) which permits unrestricted non-commercial use, distribution, and reproduction in any medium, provided the original work is properly cited.

Copyright (C) 2019The Korean Society of Pediatric Nephrology
Contrast-associated acute kidney injury (CA-AKI) is a major concern when iodinated contrast material is administered, especially in patients at risk. Efforts have been undertaken to understand the detrimental effects of contrast media (CM). With the use of low-osmolar or iso-osmolar CM the incidence of CA-AKI has steadily decreased within the past decade; however, especially in the pediatric population information is scarce. Incidence rates have been reported to range between $0 \%$ to $18.75 \%$, particularly depending on indication, selection of population (i.e. preexisting co-morbidities), and definition of AKI. Different biomarkers have been proposed, but confirmatory studies are either lacking or have contributed to their lack of diagnostic power. Proteomic approaches have been employed and may pave the way to such discovery. Prevention strategies have been tested and proposed, but the recently published AMACING and PRESERVE trials have shown that commonly used strategies (such as systematic hydration or administration of $\mathrm{N}$-acetylcysteine) have no role in the prevention of CA-AKI. We propose that thoughtful assessment of one's fluid state is the most appropriate approach and depending on the hydration status diuretics or fluid administration should be provided to achieve an euvolemic state ahead of contrast exposure.

Key words: Contrast Media, Acute Kidney Injury, Renal Insufficiency

\section{Introduction}

The incidence of acute kidney injury (AKI) in childhood is rising and its epidemiology has changed significantly over the past decades. Concurrent with technologic improvements in pediatric intensive care, a shift from primary renal causes, sepsis, and burns to renal ischemia (predominantly in the setting of cardiac surgery) and nephrotoxin exposure has been noted. Therefore, hospitalized children increasingly develop AKI not from primary kidney disease but concomitant with other systemic illness or related to treatment ${ }^{1)}$.

Over the past decades, formidable advances in radiology have greatly improved the practice of medicine, and the frequency of diagnostic and interventional imaging remains on the rise ${ }^{2}$. The use of contrast media (CM) is either indispensable in this context (e.g. coronary angiography) or greatly improves the diagnostic accuracy of the examination. Therefore, apart from radiation exposure, allergic reactions and renal toxicity remain limitations of radiological interventions.

Contrast-associated acute kidney injury (CA-AKI) refers to the sudden 
deterioration in renal function following contrast exposure in the absence of other causes. The underlying pathophysiology is incompletely understood. Etiologic factors that have been incriminated include vasoconstriction and direct tubular toxicity. CA-AKI has repeatedly been linked with adverse outcomes, such as length of hospital stay, in-hospital mortality and risk of chronic kidney disease (CKD) in later life.

Notably, causality cannot be inferred from this sequence and few studies in the field had a suitable control group to permit separation of genuine contrast-induced nephropathy from CA-AKI. Although the administration of iodinated CM is considered a major risk factor for the development of AKI, numerous recent publications have questioned the danger of modern radio-agents, and there is emerging consensus, that the risk of CA-AKI has been vastly overemphasized. Furthermore, compared with individuals with multifactorial AKI, patients with CA-AKI appear to be more likely to experience only mild decrements in kidney function that recover completely ${ }^{3)}$.

Contrast nephrotoxicity is practically non-existent in isolation and usually develops in association with concomitant insults such as hypotension, dehydration, administration of other nephrotoxins (e.g. antibiotics), and underlying comorbidities ${ }^{4,5)}$. The co-existence of several such triggers is not uncommon, particularly in the intensive care unit (ICU). Naturally, ascribing AKI to one specific cause frequently remains elusive.

Pre-existing renal disease is the greatest independent risk factor for CA-AKI; furthermore, diabetes and advanced age predispose adult patients to CA-AKI. Type and volume of CM are independent and potentially modifiable predictors for the development of CA-AKI. In the pediatric population, such predisposing factors are less well defined. Ostensibly, children might carry a lower background risk; however, they exhibit their own unique risk factors, such as prematurity and congenital organ anomalies, as discussed below.

\section{Evaluation of kidney function in children and definition of pediatric CA-AKI}

There is no gold standard for estimating the glomerular filtration rate (GFR) in children, and the limitations of serum creatinine in this context, its rate of production being proportional to muscle mass, are obvious. Although various formulas for estimating GFR exist, the Bedside Schwartz equation (estimated GFR $=[0.413 \times$ height $) /$ serum creatinine]), valid in children from 1 to 16 years, remains the most practicable method in pediatrics. In 2004, the Acute Dialysis Quality Initiative (ADQI) termed the RIFLE criteria (risk, injury, failure, loss of function, end-stage renal disease). Three years later, a pediatric modified RIFLE (pRIFLE) AKI definition was developed in critically ill children $^{6}$.

There is ongoing debate about the ideal definition of AKI in children. A new creatinine-based criterion for pediatric AKI (termed pROCK) defines AKI as a creatinine increase of both $\geq 20 \mu \mathrm{mol} / \mathrm{l}$ and $\geq 30 \%$ over the initial value within 7 days. This was tested in 102,817 Chinese children and compared to the KDIGO and pRIFLE criteria. The authors identified AKI in 5432 (5.3\%), 10,446 (10.2\%) and 15.647 (15.2\%) of participants when using the pROCK, KDIGO and the pRIFLE definitions ${ }^{7}$. Differences in the occurrence of AKI have also been reported among children with hospitalization. In line, the pRIFLE criterion found the highest rate of AKI (51.1\%) as compared to $40.3 \%$ and $37.3 \%$ with the KDIGO and AKIN criteria, respectively ${ }^{8)}$.

However, serum creatinine is an imperfect marker for the diagnosis of AKI in general and of CA-AKI in particular. Numerous novel biomarkers are being scrutinized, and candidates include neutrophil gelatinase-associated lipocalin (NGAL), kidney injury molecule-1 (KIM-1), interleukin-18 (IL-18) and liver-type fatty acid-binding protein $(\mathrm{L}-\mathrm{FABP})^{9,10)}$. For instance, Hirsch and co-workers observed substantial upregulation of urine and plasma NGAL 2 hours after $\mathrm{CM}$ in a patient population undergoing cardiac catheterization, which predicted occurrence of contrast nephropathy. 91 children were included prospectively and NGAL was measured at baseline and after 2 and 6 hours. A significant increase in urinary and plasma NGAL was observed as early as 2 hours after contrast administration in those with CA-AKI, which was defined as a $50 \%$ or greater increase in serum creatinine (11 subjects). Using a cut-off value of $100 \mathrm{ng} / \mathrm{mL}$ yielded in an excellent sensitivity, specificity and area under the receiver-operating characteristic (AUC) for the 2-hour urine NGAL (73\%, 100\%, and 
0.92) and 2-hour plasma NGAL (73\%, 100\%, and 0.91, respectively). Measurement of NGAL was found to be the most powerful predictor of childhood CA-AKI in this investigation $^{11)}$. In contrast, another study recruiting 50 children undergoing cardiac catheterization found CAAKI in 16 subjects within a period of 24 hours and reported a comparable increase in serum NGAL within the first 6 hours in both, the AKI and the non-AKI group ${ }^{12)}$.

No CA-AKI event was observed in a small study from Korea including 26 children undergoing cardiac catheterization. In line, urinary NGAL remained stable over 48 hours of follow-up, while the authors observed a steady increase of urine KIM-1, and a transient increase of L-FABP. The former increase became irrelevant when adjusted for urinary creatinine excretion. In the absence of meaningful creatinine changes, the authors concluded that L-FABP may be a useful biomarker to detect subclinical $\mathrm{AKI}^{9)}$.

A study of 123 children and adolescents with a prospective follow-up included one group $(n=58)$ receiving angiography with iopromide and one group $(n=65)$ undergoing magnetic resonance (MR) angiography/urography with gadolinium (Gd)-based CM. CA-AKI occurred in two patients in the iopromide group (3.9\%), while 9.7\% (6 patients) in the second group developed AKI. In the prediction of AKI normalized urinary KIM-1 showed the most convincing results with a AUC at 24 hours of 0.76 (sensitivity 83.3 $\%$ and specificity of $54.2 \%$ ). Other markers, such as NGAL and urinary NGAL could not predict CA-AKI accurately ${ }^{13)}$.

Ideally a biomarker predicts the risk of CA-AKI at baseline. A proteomic approach was chosen to identify such markers and ten children developed AKI within 24 hours of cardiac catheterization compared to seven without. The absence of the 41 amino acid-variant of human beta-defensin-1 yielded an AUC of 0.89-0.99 and the presence of a 4631 Da large marker had an AUC of $0.84^{14)}$. Together, both markers may provide an accurate forecast identifying children at risk to develop AKI following contrast exposure.

A prospective study of children undergoing cardiac surgery without preoperative AKI examined the differences between a creatinine-defined versus a cystatin C-defined AKI incidence. The authors found a substantially lower incidence of AKI when cystatin $\mathrm{C}$ was used to define AKI and in those with AKI interleukin-18 and KIM-1 as putative biomarkers more closely correlated with this definition ${ }^{15)}$.
Taken together, the predictive value of proposed markers of CA-AKI is controversial and more research in this direction with a larger sample size is needed. In line, uniform definition of AKI in this context is also necessary to directly compare different studies with each other. Whether a cystatin C-based definition of AKI is more informative and more closely associates with biomarkers needs to be tested in future trials.

\section{AKI in children}

AKI is a frequent comorbidity experienced by hospitalized children and exposure to nephrotoxic agents ${ }^{16)}$. Next to antibiotics such as aminoglycosides and vancomycin, $\mathrm{CM}$ ranks among the most common potential culprits prescribed to exposed individuals ${ }^{17)}$. In the ICU setting, various factors such as sepsis, cardiac dysfunction, dehydration or volume overload, may compromise renal blood flow.

It is estimated that drugs, including contrast agents, contribute to approximately $15 \%$ of all AKI cases in adults but children are usually less prone to nephrotoxicity ${ }^{18)}$.

The Nephrotoxic Injury Negated by Just-in-time Action (NINJA) recently reported a high prevalence of nephrotoxin use following congenital heart disease (CHD) surgery ${ }^{18)}$. Nephrotoxins were defined according to pre-specified criteria, and this list included CM. Although no association between high nephrotoxin exposure and AKI was observed, this was ascribed to the multitude of competitive renal insults. The authors conclude that nephrotoxin exposure may represent a modifiable risk factor for AKI in high risk individuals.

In an Israeli single center study performed on a neonatal ICU the authors demonstrated underreporting of AKI, with episodes of kidney injury often not recorded in patients' medical files ${ }^{19)}$. This is of particular concern, because over the past decade the long-term consequences of AKI have garnered increased attention and several cohort studies demonstrate that pediatric AKI is a gateway for proteinuria, hypertension, and CKD in later life. This association seems to be dose-dependent, i.e. individuals with more severe forms of AKI show a higher risk for progression to CKD. 


\section{Specific pediatric risk factors}

In recent times, the impact of prematurity on future kidney health has attracted attention, and it is now established that children born at low birth weight show an increased risk for CKD in adulthood ${ }^{20)}$. With 50,000 infants born each year at less than 28 weeks of gestation in the United States alone, the prospective impact of this trend cannot be overestimated. Reduced global nephron mass and incomplete nephrogenesis are underlying mechanisms. Subsequent postnatal events, such as infections, may further impair the development of intact tubular structures, and render individuals prone to tubular damage, when exposed to nephrotoxic agents ${ }^{20)}$.

CHD constitutes the most common congenital disorder in neonates. Critical CHD, defined as need for surgery or catheter-based intervention in the first year of life, occurs in approximately one quarter of such individuals ${ }^{21}$. Congenital anomalies of kidneys and urinary tract (CAKUT) represent another sizeable proportion of anomalies identified prenatally and are a major cause of end-stage renal disease (ESRD) in children ${ }^{22}$. For obvious reasons, both entities are associated with an increased long-term need for radiologic procedures. A vast array of other forms of congenital kidney diseases, such as idiopathic nephrotic syndrome and hereditary tubulopathies, though rare individually, amount to a substantial number of children with CKD in specialist centers. Lastly, pediatric transplant recipients are another vulnerable cohort posing unique challenges ${ }^{23)}$.

\section{Incidence of pediatric CA-AKI}

Few studies have specifically examined CA-AKI in pediatric populations. In the trial by Hwang et al., no increase in serum creatinine levels was observed in a cohort with normal kidney function, corroborating the safety of $\mathrm{CM}$ in patients without $\mathrm{CKD}^{9}$. Along similar lines, a study from a trauma center, evaluating the incidence of CA-AKI in severely injured pediatric patients $(\mathrm{n}=211)$, showed no difference in the incidence of contrast nephropathy between a non-contrast group and a group exposed to $\mathrm{CM}(8.5 \%$ vs. $7.3 \%, P$ value $=0.76)^{24)}$. Two recent trials reported incidences of pediatric CA-AKI between $4-10 \%$, but, as is frequently the case in this context, the lack of controls does not permit to draw causal inference from these results ${ }^{25}$. A study by Bedoya et al. ${ }^{26)}$, involving 140 neonates (age 0-30 days), with 59 receiving intravenous iodinated $\mathrm{CM}$, found no evidence of contrast nephrotoxicity when compared to propensity-matched control groups, not exposed to CM. Recently, McDonald et al. were able to replicate this finding in the largest cohort reported to date. Among 2,201 pediatric patients (mean age 15 years), outcomes were compared between individuals who received contrast-based computed tomography $(\mathrm{CT})$-imaging $(\mathrm{n}=1,773)$ versus patients who received non-contrast scans $(\mathrm{n}=428)$. Demographics were not balanced among the two groups, with more ICU admissions in the group receiving $\mathrm{CM}$, while pre-disposing comorbidities were more frequent in the non-contrast scan group (i.e. CKD and calcineurin inhibitor use). AKI and dialysis occurred in $3.3 \%$ and $0.1 \%$, respectively, with no significant difference between the two groups. Of note, all patients with post-CT stage $3 \mathrm{AKI}$ also had contrast-independent causes of AKI, such as hypovolemia, hypotension, and nephrotoxic medications. However, only a minority of patients ( $\mathrm{n}=145$ ) had an estimated GFR $<60 \mathrm{ml} / \mathrm{min} / 1.73$ $\mathrm{m}^{2}$, limiting the generalizability of these data ${ }^{27)}$ (see Table 1 for further details).

On the whole, the true incidence of contrast nephrotoxicity in pediatrics appears to be very low and in patients with normal kidney function, the risk of CA-AKI seems negligible.

\section{Prevention}

There are no evidence-based guidelines for CA-AKI prevention in children. In the adult population, a large number of intervention studies has been performed in this field; such studies are scarce in children. Therefore, preventive strategies in pediatrics frequently extrapolate data from the adult literature, and it can often only be assumed that such measures also provide some degree of protection for children (and do no harm). Moreover, high-quality trials with adequate follow-up and clinically meaningful endpoints beyond "delta creatinine at day 7 " have only recently started to emerge in the adult literature and frequently challenge purported standards of care. For instance, the 
benefit of preventive approaches in adults has been seriously questioned by two recent high-profile trials, namely AMACING and PRESERVE ${ }^{28,29}$; as an aside, overall AKI risk was remarkably low in both studies, despite their purported high-risk cohorts.

\section{Type of contrast}

The past four decades have seen a continuous decrease of osmolality of contrast agents, reaching physiologic levels with iso-osmolar agents (IOCM) such as iodixanol. Following a meta-analysis by Barrett and Carlisle, published in 1993, the use of hyperosmolar CM (HOCM) has largely been abandoned ${ }^{30)}$. In a large Italian cohort undergoing percutaneous coronary intervention, there were no differences in the adjusted risk of CA-AKI across 4 low-osmolar CM (LOCM), compared with iodixanol, suggesting that overall risk is low with all modern contrast agents ${ }^{31,32)}$. In line, Zo'o and colleagues performed a trial comparing the LOCM iobitrol with iodixanol. 128 children (age 8.7 \pm 4.7 years) undergoing $\mathrm{CT}$ were randomized and no significant difference of AKI was found in this low risk cohort ${ }^{25)}$.

\section{Volume of contrast}

The amount of CM used is frequently cited as an independent predictor for the development of contrast nephropathy. However, according to the recent manual of the American College of Radiology (ACR), there is a lack of robust data to support a dose-toxicity relationship for intravenous (IV) iodinated CM, with only intra-arterial CM showing a directly proportional relationship ${ }^{33}$. Very small volumes of contrast agents, typically $1.5-2 \mathrm{ml} / \mathrm{kg}$, are usu- ally administered in neonates and infants ${ }^{34)}$, and, as stated in a previous review, the "volume of contrast should be determined by the size of the child, the imaging study being performed, and the estimated GFR" ${ }^{35}$. Nevertheless, it seems prudent to limit contrast volume in at-risk individuals, provided that the quality of diagnostic imaging is not jeopardized. An ALARA (as low as reasonably achievable) concept is usually recommended.

\section{Route of administration}

The majority of data in the field of contrast nephropathy stems from cardiology studies. Unlike IV contrast administration, in coronary angiography the injection is intraarterial and supra-renal. Cholesterol emboli, dislodged by the catheter, are possible competitor strikes, and the kidneys are exposed to a more abrupt and concentrated $\mathrm{CM}$ dose. Consequently, a higher overall incidence of CA-AKI is usually found in studies of cardiac angiography than in studies involving IV contrast administration. However, McDonald et al. recently reported a similar risk of AKI after intra-arterial and IV administration of CM (9.9\% versus $11 \%$ ) in a large cohort of patients that received both routes of administration ${ }^{36)}$. Despite this, patients undergoing such intra-arterial procedures should be monitored with particular attention, because intra-arterial angiography is associated with a higher risk of post-contrast AKI, regardless of etiology.

\section{Hydration}

Fluid administration increases urine flow rate, reduces $\mathrm{CM}$ concentration in the tubule and expedites CM elimi-

Table 1. Studies of Contrast-associated Acute Kidney Injury in Children. Studies Showed a Wide Range of Incidence Rates, which is in Analogue with Adult Data. Among other Factors this Range is Clearly Influenced by Diverse Criteria used to Define Acute Kidney Injury

\begin{tabular}{|c|c|c|c|c|c|c|c|c|}
\hline Author & $\begin{array}{c}\text { Year of } \\
\text { publication }\end{array}$ & $\begin{array}{c}\mathrm{N} \\
\text { (iodinated CM) }\end{array}$ & Type of study & $\begin{array}{l}\text { Control group } \\
\text { propensity }\end{array}$ & $\begin{array}{l}\text { Age } \\
\text { (years) }\end{array}$ & Intervention & AKI definition & Incidence \\
\hline Ajami $^{48)}$ & 2010 & 80 & prospective & no & $<18$ & CA & RIFLE & $18.75 \%$ \\
\hline$Z^{\prime} \mathrm{O}^{24)}$ & 2011 & 145 & prospective & $\begin{array}{l}\text { iodixanol vs. } \\
\text { iobitridol }\end{array}$ & $\leq 16$ & $\mathrm{CT}$ & $\begin{array}{l}\mathrm{CrCl} \text { changes; } \mathrm{CA}-\mathrm{AKI} \\
(<25 \% \text { reduction in } \mathrm{CrCl})\end{array}$ & $\begin{array}{l}4.8 \% \text { (iobitridol), } \\
10.6 \% \text { (iodixanol) }\end{array}$ \\
\hline Hwang ${ }^{8)}$ & 2014 & 26 & prospective & no & 7.1 & CA & - & $0 \%$ \\
\hline Cantais $^{49)}$ & 2016 & 346 & retrospective, cohort & no & $<16$ & $\mathrm{CT}$ & KDIGO & $10.3 \%$ \\
\hline McDonald ${ }^{26)}$ & 2018 & $2,201(1,391)$ & retrospective, cohort & yes & $<18$ & $\mathrm{CT}$ & KDIGO & $\begin{array}{l}3.5 \% \text { (contrast), } \\
7.8 \% \text { (no contrast) }\end{array}$ \\
\hline Paul $^{23)}$ & 2019 & 211 & retrospective & $\begin{array}{l}\text { Contrast versus } \\
\text { no } C T \text { contrast }\end{array}$ & $<15$ & $\mathrm{CT}$ & AKIN & $\begin{array}{l}7.3 \% \text { (contrast), } \\
8.5 \% \text { (no contrast) }\end{array}$ \\
\hline
\end{tabular}

Abbreviations: CM (contrast media), CA (coronary angiography), CT (computed tomography), RIFLE (risk, injury, failure, loss, ESRD), CrCl (creatinine clearance), CA-AKI (contrast-associated acute kidney injury), KDIGO (Kidney Disease Improving Global Outcomes), AKIN (Acute Kidney Injury Network). 
www.chikd.org

nation, thereby limiting exposure time of tubular cells to the deleterious effects of CM. Consequently, intravenous hydration is considered standard of care for patients at risk for CA-AKI, with $0.9 \%$ normal saline (NS) being the most common utilized fluid. However, in the AMACING trial ${ }^{28)}$, involving 660 adult patients with CKD stage 3, preventive hydration with NS not only failed to show benefit in terms of AKI risk but reported 13 episodes (4\%) of symptomatic heart failure in the treatment group versus none in the control group. Along similar lines, Liu et al. observed an increased risk of CA-AKI and death in patients with CKD with "excessively high hydration", as calculated by hydration volume to weight ${ }^{37}$. It seems obvious that children at increased risk for contrast nephropathy, such as patients with CHD, are also particularly prone to volume overload, so a cautionary approach regarding hydration is mandatory.

Limiting exposure of the kidney to radiocontrast by generating high urine flow without causing hypovolemia ("forced diuresis") remains an accepted tool in preventing CA-AKI; this concept is also cited in the pediatric literature ${ }^{35)}$. In adults, the P.R.I.N.C.E. study ${ }^{38)}$, published in 1999, provided evidence that "good urine output" (urine flow rates above $150 \mathrm{ml} / \mathrm{h}$ ) following contrast exposure is associated with a reduced risk of AKI. The study involved 98 patients with CKD stage 3-4 (median estimated creatinine clearance of $32 \mathrm{ml} / \mathrm{min}$ ) undergoing elective coronary angiography. In hindsight, assessment of volume status in this study appears unduly invasive, with right heart catheterization performed in all participants, and Swan-Ganz catheterization in individuals randomized to the experimental arm; moreover, all patients had urinary catheters placed. Therefore, the practicality of this frequently cited paper seems questionable. The RenalGuard ${ }^{\oplus}$ system, a device delivering replacement solution in real-time and in an amount matched to urinary output, thereby maintaining euvolemia, is a relatively new strategy for CA-AKI prevention. It also involves placement of a urinary catheter; price issues and lack of data in children remain limitations ${ }^{39)}$.

For all hydration strategies, the optimal type, quantity, timing, and duration of fluid administration remain largely unresolved issues and well-defined protocols are lacking, especially for children.

\section{Pharmacologic strategies}

\section{No approved drugs exist for the prevention of CA-} AKI.

$\mathrm{N}$-Acetylcysteine (NAC), a free radical scavenger antioxidant, arguably represents the best-known agent in the field of CA-AKI prevention. The landmark paper by Tepel et al. ${ }^{40)}$ sparked a flurry of studies, which have been subject to a number of meta-analyses. Due to the heterogeneity of study designs, it was difficult to draw clear conclusions. Following the publication of the ACT trial and the PRESERVE study ${ }^{29,41)}$, both reporting no benefit of NAC in adult patients with CKD, this compound has lost much of its appeal. There are no publications pertaining to its use in the field of pediatric CA-AKI. Some experts continue to use this agent in conjunction with hydration in children deemed at high risk for contrast nephropathy, although they acknowledge that this approach is not backed by the available literature, and its routine for AKI prophylaxis is not recommended. Notably, NAC should not be seen as a substitute for appropriate pre-procedural patient screening and other measures, as outlined below.

Acetazolamide, a carbonic anhydrase inhibitor, has been proposed to be beneficial in the prevention of CA-AKI. One study involving 96 pediatric patients with CKD (aged 3 to 19 years, mean creatinine clearance $52 \mathrm{ml} / \mathrm{min} / 1.73 \mathrm{~m}^{2}$ ), who underwent coronary angiograms, $\mathrm{CT}$, or renal angiograms, found that urinary alkalinization with acetazolamide resulted in a lower incidence of postcontrast $\mathrm{AKI}$ than intravenous sodium bicarbonate ( 0.0 versus $8.7 \% ; P=0,049$ ) ${ }^{42)}$. However, the difference in mean serum creatinine $(1.32 \pm 0.13 \mathrm{mg} / \mathrm{dL}$ versus $1.39 \pm 0.14 \mathrm{mg} / \mathrm{dL}) 48$ hours after contrast exposure, although statistically significant, is of doubtful clinical relevance. No corroborating evidence in pediatric cohorts has emerged since publication of this trial.

The alpha-2-receptor agonist dexmedetomidine is approved for sedation in adult ICU patients, but is increasingly used off-label in the pediatric setting for various indications ${ }^{43}$. Its low risk of respiratory depression makes it a comparatively safe agent. In a murine model, alpha-2-receptor agonists exhibited renal protective effects following iohexol injection ${ }^{44)}$. Attenuation of contrast-mediated renal blood reduction after radiocontrast injection is a postulated mechanism ${ }^{44}$. The use of dexmedetomidine for the pre- 
vention of AKI of diverse etiologies, including CA-AKI, has been championed by a Turkish group. Bayram et al. ${ }^{45}$ analyzed the effect of dexmedetomidine in a randomized, controlled trial (RCT) involving 60 children with CHD, scheduled for coronary angiography. The authors observed CA-AKI (pRIFLE criteria) in 3 patients (10\%) in the intervention arm and in 11 (36.7\%) in the control arm. Moreover, renin and NGAL levels at 24 hours after angiography were significantly lower in the dexmedetomidine group. Although bradycardia was noted in 7 patients in the active arm as compared to 3 in the control group, this did not reach statistical significance. A recent meta-analysis of pharmacological interventions for AKI prevention after pediatric cardiac surgery reported a lower incidence of AKI in the dexmedetomidine groups (OR 0.65 ${ }^{46)}$. Future trials should clarify the effectiveness of this agent for the prevention of CA-AKI and it remains to be seen, whether this drug will stand the test of time.

Numerous other agents have been evaluated, without showing benefit beyond single center trials ${ }^{35}$.

Given that multiple lines of evidence suggest that no single intervention strategy may successfully prevent CAAKI, a multimodal approach for renoprotection has been advocated ${ }^{47)}$. We propose that currently physicians should identify at-risk groups, hold potentially nephrotoxic drugs prior to contrast administration, approach the interventiologist preemptively with a view to minimizing contrast volume, and target euvolemia.

Once CA-AKI has occurred, management follows general recommendations for AKI; in this context, it is important to avoid "second hits" i.e. repeat CM administra$\operatorname{tion}^{48)}$.

\section{CA-AKI: Much ado about little?}

CA-AKI is widely cited as a leading cause of in-hospital $A K I$ and its validity remains a clinical dogma. Newhouse et al. ${ }^{49)}$ were the first to observe that patients who were not exposed to iodinated contrast showed rates of AKI similar to patients with contrast nephropathy after CT. These authors showed that controlling for potentially confounding clinical conditions that may trigger AKI is difficult and that a "post hoc, ergo propter hoc" reasoning has been in place for too long. Several recent publications ${ }^{50,51)}$, using propensity score-matching, have further challenged the doctrine of CA-AKI as a stand-alone entity. Moreover, in hospitalized individuals, random changes in serum creatinine ('background noise') may result in AKI misclassification, irrespective of CM being present. Patients who receive contrast often have numerous other independent risk factors for AKI, either from their primary pathology or comorbidities, independent of contrast administration. As stated by Ehrmann et al. ${ }^{52)}$, this distinction between a causal and an associative relationship between $\mathrm{CM}$ and $\mathrm{AKI}$ is of great importance, because suboptimal care may result from excessive caution ("renalism"). For fear of nephrotoxicity, patients may undergo unenhanced imaging which is less accurate than contrast-enhanced imaging, resulting in negative impact on clinical decisions related to treatment. Lastly, the bulk of negative trials in the field of CA-AKI prevention could be regarded as another piece in the chain of evidence indicating that contrast nephropathy may be more fiction than fact.

\section{Conclusion}

In the absence of randomized controlled trials, widespread lack of meaningful endpoints, and with only a handful of good quality papers available, it is difficult to make firm recommendations. Likewise, in view of the growing body of literature, the search for a single panacea to prevent contrast nephropathy in general and in pediatrics in particular will likely remain futile. Children at risk for AKI should be treated with due diligence, regardless of whether CA-AKI is interpreted as contrast-induced AKI (i.e. a causative diagnosis) or post-contrast AKI (a correlative diagnosis). This should entail a multimodal approach aimed at AKI prevention, close cooperation between care providers, and early (preemptive) nephrology consultation.

\section{Conflict of interest}

Both authors declare no competing conflicts of interest related to the submitted work. 


\section{ORCID}

Andreas Kronbichler https://orcid.org/0000-00022945-2946

\section{References}

1. Goldstein SL. Acute kidney injury in children and its potential consequences in adulthood. Blood Purif 2012;33:131-7.

2. Katzberg RW, Haller C. Contrast-induced nephrotoxicity: clinical landscape. Kidney Int Suppl 2006:S3-7.

3. Tandukar S, Rondon-Berrios H, Weisbord SD. Intravascular lodinated Contrast Is an Independent Cause of Acute Kidney Injury Following Coronary Angiography. South Med J 2019;112:541-6.

4. Windpess I M, Kronbichler A. Pro: Contrast-induced nephropathyshould we try to avoid contrast media in patients with chronic kidney disease? Nephrol Dial Transplant 2018;331317-9.

5. Ewing MJ, Eidt JF. Con: Contrast-induced nephropathy-should we try to avoid contrast media in patients with chronic kidney disease? Nephrol Dial Transplant 2018;33:1320-2.

6. Akcan-Arikan A, Zappitelli M, Loftis LL, Washburn KK, Jefferson LS, Goldstein SL. Modified RIFLE criteria in critically ill children with acute kidney injury. Kidney Int 2007;71:1028-35.

7. Xu X, Nie S, Zhang A, Jianhua M, Liu HP, Xia H, et al. A New Criterion for Pediatric AKI Based on the Reference Change Value of Serum Creatinine. J Am Soc Nephrol 2018;29:2432-42.

8. Sutherland SM, Byrnes JJ, Kothari M, Longhurst CA, Dutta S, Garcia P, et al. AKI in hospitalized children: comparing the pRIFLE, AKIN, and KDIGO definitions. Clin J Am Soc Nephrol 2015;10:554-61.

9. Hwang YJ, Hyun MC, Choi BS, Chun SY, Cho MH. Acute kidney injury after using contrast during cardiac catheterization in children with heart disease. J Korean Med Sci 2014;29:1102-7.

10. Yim HE. Neutrophil Gelatinase-Associated Lipocalin and Kidney Diseases. Child Kidney Dis 2015;19:79-88.

11. Hirsch R, Dent C, Pfriem H, Allen J, Beekman RH, 3rd, Ma Q, et al. NGAL is an early predictive biomarker of contrast-induced nephropathy in children. Pediatr Nephrol 2007;22:2089-95.

12. Tkaczyk M, Tomczyk D, Jander A, Goreczny S, Moszura T, Dryzek P, et al. Glomerular filtration decrease after diagnostic cardiac catheterisation in children with congenital cardiac malformation - the role of serum creatinine, cystatin C, neutrophil gelatinase and urine output monitoring. Postepy Kardiol Interwencyjnej 2018;14:67-74.

13. Spasojevic-Dimitrijeva B, Kotur-Stevuljevic J, Dukic M, Paripovic D, Milosevski-Lomic G, Spasojevic-Kalimanovska V, et al. Serum Neutrophil Gelatinase-Associated Lipocalin and Urinary Kidney Injury Molecule-1 as Potential Biomarkers of Subclinical Nephrotoxicity After Gadolinium-Based and lodinated-Based Contrast Media Exposure in Pediatric Patients with Normal Kidney Func- tion. Med Sci Monit 2017;23:4299-305.

14. Bennett MR, Ravipati N, Ross G, Nguyen MT, Hirsch R, Beekman $\mathrm{RH}$, et al. Using proteomics to identify preprocedural risk factors for contrast induced nephropathy. Proteomics Clin Appl 2008;2: 1058-64.

15. Zappitelli M, Greenberg JH, Coca SG, Krawczeski CD, Li S, ThiessenPhilbrook HR, et al. Association of definition of acute kidney injury by cystatin C rise with biomarkers and clinical outcomes in children undergoing cardiac surgery. JAMA Pediatr 2015;169: 583-91.

16. Uber AM, Montez-Rath ME, Kwiatkowski DM, Krawczeski CD, Sutherland SM. Nephrotoxin exposure and acute kidney injury in critically ill children undergoing congenital cardiac surgery. Pediatr Nephrol 2018;33:2193-9.

17. Goldstein SL, Mottes T, Simpson K, Barclay C, Muething S, Haslam $D B$, et al. A sustained quality improvement program reduces nephrotoxic medication-associated acute kidney injury. Kidney Int 2016;90:212-21.

18. Uber AM, Sutherland SM. Acute kidney injury in hospitalized children: consequences and outcomes. Pediatr Nephrol 2018.

19. Cleper R, Shavit I, Blumenthal D, Reisman L, Pomeranz G, Haham $A$, et al. Neonatal acute kidney injury: recording rate, course, and outcome: one center experience. J Matern Fetal Neonatal Med 2019;32:3379-85.

20. Starr MC, Hingorani SR. Prematurity and future kidney health: the growing risk of chronic kidney disease. Curr Opin Pediatr 2018; 30:228-35.

21. Oster ME, Lee KA, Honein MA, Riehle-Colarusso T, Shin M, Correa A. Temporal trends in survival among infants with critical congenital heart defects. Pediatrics 2013;131:e1502-8.

22. Seikaly MG, Ho PL, Emmett L, Fine RN, Tejani A. Chronic renal insufficiency in children: the 2001 Annual Report of the NAPRTCS. Pediatr Nephrol 2003;18:796-804.

23. Abbas FM, Julie BM, Sharma A, Halawa A. "Contrast nephropathy" in renal transplantation: Is it real? World J Transplant 2016;6:6828.

24. Paul KM, 2nd, Johnson J, Garwe T, Sarwar Z, Motghare P, Daly W, et al. Computed Tomography with Intravenous Contrast Is Not Associated with Development of Acute Kidney Injury in Severely Injured Pediatric Patients. Am Surg 2019;85:e1-e5.

25. Zo'o M, Hoermann M, Balassy C, Brunelle F, Azoulay R, Pariente D, et al. Renal safety in pediatric imaging: randomized, doubleblind phase IV clinical trial of iobitridol 300 versus iodixanol 270 in multidetector CT. Pediatr Radiol 2011;41:1393-400.

26. Ciavatta DJ, Yang J, Preston GA, Badhwar AK, Xiao H, Hewins P, et al. Epigenetic basis for aberrant upregulation of autoantigen genes in humans with ANCA vasculitis. J Clin Invest 2010;120: 3209-19.

27. McDonald JS, McDonald RJ, Tran CL, Kolbe AB, Williamson EE, Kallmes DF. Postcontrast Acute Kidney Injury in Pediatric Patients: A Cohort Study. Am J Kidney Dis 2018;72:811-8.

28. Nijssen EC, Rennenberg RJ, Nelemans PJ, Essers BA, Janssen MM, 
Vermeeren MA, et al. Prophylactic hydration to protect renal function from intravascular iodinated contrast material in patients at high risk of contrast-induced nephropathy (AMACING): a prospective, randomised, phase 3, controlled, open-label, noninferiority trial. Lancet 2017;389:1312-22.

29. Weisbord SD, Gallagher M, Jneid H, Garcia S, Cass A, Thwin SS, et al. Outcomes after Angiography with Sodium Bicarbonate and Acetylcysteine. N Engl J Med 2018;378:603-14.

30. Barrett BJ, Carlisle EJ. Metaanalysis of the relative nephrotoxicity of high- and low-osmolality iodinated contrast media. Radiology 1993;188:171-8.

31. Azzalini L, Vilca LM, Lombardo F, Poletti E, Laricchia A, Beneduce A, et al. Incidence of contrast-induced acute kidney injury in a large cohort of all-comers undergoing percutaneous coronary intervention: Comparison of five contrast media. Int J Cardiol 2018;273:69-73.

32. Kronbichler A, Shin Jl, Windpessl M. What is left to prevent contrast-induced acute kidney injury? No difference between low and iso-osmolar contrast media. Int J Cardiol 2018;273:94-5.

33. Media ACoDaC. ACR Manual On Contrast Media. 2018.

34. Trout AT, Dillman JR, Ellis JH, Cohan RH, Strouse PJ. Patterns of intravenous contrast material use and corticosteroid premedication in children--a survey of Society of Chairs of Radiology in Children's Hospitals (SCORCH) member institutions. Pediatr Radiol 2011:41:1272-83.

35. Verghese PS. Contrast nephropathy in children. J Pediatr Intensive Care 2014;3:45-52.

36. McDonald JS, Leake CB, McDonald RJ, Gulati R, Katzberg RW, Williamson EE, et al. Acute Kidney Injury After Intravenous Versus Intra-Arterial Contrast Material Administration in a Paired Cohort. Invest Radiol 2016;51:804-9.

37. Liu Y, Li H, Chen S, Chen J, Tan N, Zhou Y, et al. Excessively High Hydration Volume May Not Be Associated With Decreased Risk of Contrast-Induced Acute Kidney Injury After Percutaneous Coronary Intervention in Patients With Renal Insufficiency. J Am Heart Assoc 2016;5.

38. Stevens MA, McCullough PA, Tobin KJ, Speck JP, Westveer DC, Guido-Allen DA, et al. A prospective randomized trial of prevention measures in patients at high risk for contrast nephropathy: results of the P.R.I.N.C.E. Study. Prevention of Radiocontrast Induced Nephropathy Clinical Evaluation. J Am Coll Cardiol 1999; 33:403-11.

39. Mattathil S, Ghumman S, Weinerman J, Prasad A. Use of the RenalGuard system to prevent contrast-induced AKI: A metaanalysis. J Interv Cardiol 2017;30:480-7.
40. Tepel M, van der Giet M, Schwarzfeld C, Laufer U, Liermann D, Zidek W. Prevention of radiographic-contrast-agent-induced reductions in renal function by acetylcysteine. $\mathrm{N}$ Engl J Med 2000;343:180-4.

41. Investigators ACT. Acetylcysteine for prevention of renal outcomes in patients undergoing coronary and peripheral vascular angiography: main results from the randomized Acetylcysteine for Contrast-induced nephropathy Trial (ACT). Circulation 2011; 124:1250-9.

42. Assadi F. Acetazolamide for prevention of contrast-induced nephropathy: a new use for an old drug. Pediatr Cardiol 2006;27: 238-42.

43. Phan $\mathrm{H}$, Nahata MC. Clinical uses of dexmedetomidine in pediatric patients. Paediatr Drugs 2008;10:49-69.

44. Billings FTt, Chen SW, Kim M, Park SW, Song JH, Wang S, et al. alpha2-Adrenergic agonists protect against radiocontrastinduced nephropathy in mice. Am J Physiol Renal Physiol 2008; 295:F741-8.

45. Bayram A, Ulgey A, Baykan A, Narin N, Narin F, Esmaoglu A, et al. The effects of dexmedetomidine on early stage renal functions in pediatric patients undergoing cardiac angiography using nonionic contrast media: a double-blind, randomized clinical trial. Paediatr Anaesth 2014;24:426-32.

46. Liu Y, Sheng B, Wang S, Lu F, Zhen J, Chen W. Dexmedetomidine prevents acute kidney injury after adult cardiac surgery: a metaanalysis of randomized controlled trials. BMC Anesthesiol 2018; $18: 7$.

47. McCullough PA. Multimodality prevention of contrast-induced acute kidney injury. Am J Kidney Dis 2008;51:169-72.

48. Lakhal K, Ehrmann S, Robert-Edan V. Iodinated Contrast Medium Renal Toxicity: The Phantom Menace or Much Ado About Nothing? Crit Care Med 2017;45:e745-e6.

49. Newhouse JH, Kho D, Rao QA, Starren J. Frequency of serum creatinine changes in the absence of iodinated contrast material: implications for studies of contrast nephrotoxicity. AJR Am J Roentgenol 2008;191:376-82.

50. McDonald JS, McDonald RJ, Williamson EE, Kallmes DF, Kashani K. Post-contrast acute kidney injury in intensive care unit patients: a propensity score-adjusted study. Intensive Care Med 2017;43: 774-84.

51. McDonald JS, McDonald RJ, Williamson EE, Kallmes DF. Is Intravenous Administration of lodixanol Associated with Increased Risk of Acute Kidney Injury, Dialysis, or Mortality? A Propensity Score-adjusted Study. Radiology 2017;285:414-24.

52. Ehrmann S, Aronson D, Hinson JS. Contrast-associated acute kidney injury is a myth: Yes. Intensive Care Med 2018;44:104-6. 